\title{
Research Trends in General Medicine Departments of University Hospitals in Japan
}

This article was published in the following Dove Press journal:

International Journal of General Medicine

\author{
Takashi Watari ${ }^{1,2, *}$ \\ Masaki Tago $\mathbb{D}^{3, *}$ \\ Kiyoshi Shikino $\mathbb{1}^{4}$ \\ Shun Yamashita $\mathbb{D D}^{3}$ \\ Naoko E Katsuki ${ }^{3}$ \\ Motoshi Fujiwara ${ }^{3}$ \\ Shu-ichi Yamashita ${ }^{3}$ \\ 'General Medicine Center, Shimane \\ University Hospital, Shimane, Japan; \\ ${ }^{2}$ Master of Healthcare Quality and Patient \\ Safety, Harvard Medical School, Boston, \\ MA, USA; ${ }^{3}$ Department of General \\ Medicine, Saga University Hospital, Saga, \\ Japan; ${ }^{4}$ Department of General Medicine, \\ Chiba University Hospital, Chiba, Japan \\ *These authors contributed equally to \\ this work
}

Correspondence: Masaki Tago Department of General Medicine, Saga University Hospital, 5-I-I Nabeshima,

Saga, 849-850I, Japan

$\mathrm{Tel}+81952343238$

Fax +81952342029

Email tagomas@cc.saga-u.ac.jp
Purpose: The training of generalist physicians in university hospitals needs to emphasize development of their research role in order to continue improving their research capacity and their standing in academic hospitals in Japan. This cross-sectional descriptive study aimed to survey departments of general medicine (GM) in university hospitals in Japan to identify the research areas and themes pursued by academic generalist physicians.

Patients and Methods: The heads of the departments of GM from 71 university hospitals in Japan were enrolled. The main outcomes studied were the identification of the main research areas and themes in academic departments of GM, based on the classification of the National Grants-in-Aid for Scientific Research (KAKENHI): clinical research, public health, preventive medicine, medical education, basic science, health services and safety and quality. Results: We received 47 of 71 replies $(66.2 \%$ response rate). Clinical research was the most common area of research $(62 \%)$, followed by public health and preventive medicine $(14 \%)$, medical education (11\%), and basic sciences (9\%). Only one department identified health services and safety and quality as a research area $(2 \%)$. There was marked variability in research areas across the different departments, with $23 \%$ of the research targeting the highest specialties, particularly organ-specific research in the fields of gastroenterology, cardiology, immunology, neurology, metabolic endocrinology, and hematology-oncology.

Conclusion: The training of generalist physicians in university hospitals needs to emphasize development of their research role in order to continue improving the research capacity and the standing generalist physicians in academic hospitals in Japan.

Keywords: general medicine, research, academic activity

\section{Introduction}

It is essential that generalist physicians be actively involved in research to improve patient care quality, promote patient-centered care, and, importantly, develop and promote general medicine (GM) as an academic discipline. However, internationally, the contribution of generalist physicians to research is not as high as that of other subspecialists. ${ }^{1-3}$ The field of practice of generalist physicians has been classified into family, general, and hospital medicine, with the definition and classification varying between countries and different healthcare systems. ${ }^{3-5}$ In Japan, the generalist physician role was established as one of the 19 medical board subspecialties only in $2018 .{ }^{6}$ The role of generalist physicians in Japan is varied, including hospital medicine, family medicine, outpatient management of difficult diagnostic cases, or a mix of these roles. Therefore, the definition and actual role of generalists within Japan's unique healthcare system has not yet been clearly established, ${ }^{7}$ with generalists adapting their role depending on the needs of clinical settings in which they work. This has resulted in 
difficulty for generalist physicians to become active in research in university hospitals. ${ }^{5,8}$ Wachter and Goldman ${ }^{3}$ identified the burden of teaching as a potential barrier to generalists in becoming involved in research. Specifically, the clinical success of generalists has led to a large increase in their recruitment in hospitals, with a large focus of their contribution being to teaching in academic hospitals. With regard to research, generalists contribute to clinical research, health services, quality, and safety and clinical education research, all of which require a cross-sectional and comprehensive perspective which aligns with their training. ${ }^{89}$ Through research, generalists have provided evidence to support the benefits and safety of their practice. ${ }^{10}$ However, in Japan, the research involvement of generalist physicians has not been clearly defined. Therefore, our aim was to survey departments of GM in university hospitals in Japan to identify the research areas and themes pursued by academic generalist physicians. This is an important issue if we are to better describe the roles and contributions of generalist physicians in Japan.

\section{Methods}

This was a cross-sectional descriptive study of all GM departments in the 71 university hospitals in Japan (Tertiary hospital). Our survey was distributed by the Council of Japanese university hospitals, between June 1 and July 17, 2020. The survey was conducted among department heads for accurate documentation of the main research area in their department, defined based on the classification of the National Grants-in-Aid for Scientific Research (KAKENHI), ${ }^{11}$ as follows: clinical research, public health, preventive medicine, medical education, basic science, health services and safety and quality. The data were collected through an online platform. Surveys returned with incomplete information or inappropriate descriptions were excluded from our analysis.

Ethics approval was not required owing to our use of surveys; it was made clear at the start of the survey that no personal information was being collected and that university data would be anonymized.

\section{Results}

Complete surveys were returned by 47 of the 71 departments surveyed (66.2\% response rate). Of these 47 participating departments, 31 were affiliated with public medical schools and the other 16 with private medical schools. The academic position of those responsible for completing this survey was as follows: Professor $(n=35)$, Associate Professor $(n=4)$,
Lecturer ( $n=6)$, Assistant Professor $(n=2)$. The distribution of the type of research undertaken by generalist physicians is shown in Figure 1. Clinical research was the most common area of research $(62 \%)$, followed by public health and preventive medicine (14\%), medical education (11\%), and basic sciences (9\%). Only one department identified health services, safety, and quality as a research area $(2 \%)$. Note, the research area for one center was not specified $(2 \%)$. The specific research themes, as per the KAKENHI classification, are reported in Table 1. On average, one department of GM reported being involved in 1.5 research themes, with research in general internal medicine being the most common $(n=20)$, followed by medical management and medical sociology $(n=11)$, and infectious disease medicine $(n=5)$. There was marked variability in the areas of research across the different departments, with $23 \%$ of the research targeting the highest specialties, particularly organ-specific research in the fields of gastroenterology, cardiology, immunology, neurology, metabolic endocrinology, and hematology-oncology.

\section{Discussion}

This was a cross-sectional survey of the areas and themes of research undertaken in departments of GM of university hospitals in Japan. As previously stated, understanding the research areas of generalist physicians is important to better define their role within academic hospitals, particularly in Japan where the generalist subspecialty is new and not yet well defined in term of scope. The role of generalist physicians has been better defined in European countries and in the United States, where generalists are regrouped under the umbrella terms of family medicine, general internal medicine, and hospital medicine..$^{3-5,12}$ The need to better define the role of generalists is essential for appropriate allocation of medical resources, considering the different and often competing,

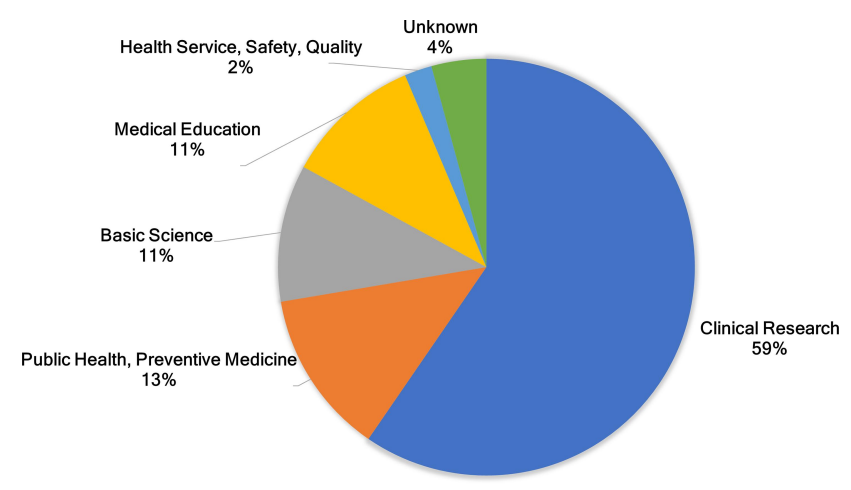

Figure I Types of research fields identified by the General Medicine departments in our 2020 survey. 
Table I Research themes of General Medicine departments of university hospitals classified using the Review Section of the Japanese KAKENHI classification

\begin{tabular}{|c|c|c|}
\hline $\begin{array}{l}\text { Section } \\
\text { Number* }\end{array}$ & Basic Section & $\mathbf{n}$ \\
\hline 52010 & General internal medicine & 20 \\
\hline 58010 & Medical management and medical sociology & $\mathrm{II}$ \\
\hline 54030 & Infectious disease medicine & 5 \\
\hline 53010 & Gastroenterology & 4 \\
\hline 58030 & $\begin{array}{l}\text { Hygiene and public health-related: excluding } \\
\text { laboratory approach }\end{array}$ & 4 \\
\hline 53020 & Cardiology & 3 \\
\hline 54040 & Metabolism and endocrinology & 3 \\
\hline 49070 & Immunology & 2 \\
\hline 55060 & Emergency medicine & 2 \\
\hline 58020 & $\begin{array}{l}\text { Hygiene and public health-related: including } \\
\text { laboratory approach }\end{array}$ & 2 \\
\hline 58080 & $\begin{array}{l}\text { Gerontological nursing and community health } \\
\text { nursing }\end{array}$ & 2 \\
\hline 10020 & Educational psychology & I \\
\hline 50020 & Tumor diagnostics and therapeutics & $\mathrm{I}$ \\
\hline 52020 & Neurology & $\mathrm{I}$ \\
\hline 53030 & Respiratory medicine & I \\
\hline 54010 & Hematology and medical oncology & $\mathrm{I}$ \\
\hline 59010 & Rehabilitation science & I \\
\hline 59040 & Nutrition science and health science & I \\
\hline 90130 & Medical systems & I \\
\hline 90140 & Medical technology assessment & I \\
\hline 08020 & Social welfare & I \\
\hline 09050 & Tertiary education & I \\
\hline
\end{tabular}

Note: *Classification number of the Review Section of the Japanese KAKENHI.

demands within medical systems, and to appropriately address the barriers limiting the contribution of generalists to research in university hospitals. ${ }^{3,8,9,12}$ A previous report on the 25 -year history of generalist physicians in the United States ${ }^{12}$ identified the following key factors as important for generalists to define their relevance and standing in hospitals: exerting institutional influence and prestige; faculty development; assuming leadership roles in medicine; defined clinical responsibilities; financial stability of GM departments; research productivity; and developing the field as an academic discipline. In the United States, GM has achieved its academic status through general physicians' significant contributions to clinical practice and medical education. ${ }^{1,13,14}$ On the other hand, several studies show that research contribution by GM physicians has been limited. $^{13,14}$ The GM departments of university hospital are expected to contribute to research, in addition to the above stated contribution to practice and education, to enhance the role and value of GM departments. ${ }^{15}$ With regard to research, the research interests of hospitalists is generally in clinical research, health services, quality improvement, and education. ${ }^{5,12}$ However, traditionally, the research direction for Departments of GM in hospitals has been in basic biomedical research. Yet, the area of research for generalists in hospitals is rarely in basic laboratory medicine, ${ }^{8}$ which may pose a further barrier to generalists in establishing a program of research in their specialty. As the medical training of generalists intersects with various medical subspecialties, generalists are well-positioned to contribute to research on health services, quality, and safety; health information, eg diagnostic error, patient safety, quality improvement, and clinical education. ${ }^{7}$

In Japan, generalist medicine physicians have an overall low contribution to research, with only $4.7 \%$ of the research presented at the Japan Primary Care Association, the largest society for general practitioners in Japan, being ultimately published in a peer-review journal, a rate that is noticeably lower than that of other medical departments. ${ }^{15}$ Two issues are important to consider in this regard. First, as the heads of GM departments are often from various internal medicine specialties, the focus on basic sciences research is likely to persist. ${ }^{7,14,15}$ The second issue regards mentorship which is important to support generalists in developing a program of research; however, as the generalist program became a medical board subspecialty in Japan only in 2018, the generalist subspecialty is still in its developmental stage and, thus, mentorship for research may not be readily available. $7,8,11,14$

The limitations of our study need to be acknowledged. Foremost is the possibility of a selection bias as we did not obtain responses from all university hospitals. In addition, we note that identified areas of research on our survey may not align with the allocation of research funds. Without information on funding, it is not possible at this time to fully compare the research capacity of generalists in Japan to that in other countries. The results of this survey may also be influenced by the field of study and practice of the principal author. Moreover, in Japan, national universities are regrouped under the umbrella of one university hospital and chair, with only 
some private universities having a chair in some hospital branches. This organization would influence the range of research conducted by Departments of GM in Japan.

\section{Conclusion}

This is the first study to report on the current research interests and trends in the departments of GM at university hospitals in Japan. Based on our findings and considering previous reports, we believe that research needs to be emphasized within the role of generalists in university hospitals in order to continue improving the contribution of standing generalists in academic hospitals in Japan. Previous findings from the United States may help in this regard, providing evidence that generalist physicians are well-positioned to contribute to research on health services, quality, and safety; health information; and clinical education.

\section{Acknowledgments}

We thank Dr. Taro Shimizu, Dokkyo Medical University, Dr. Yosuke Sasaki, Toho University School of Medicine, and Dr. Hiromizu Takahashi, Juntendo University for their assistance in developing the survey.

\section{Disclosure}

T.W. is supported by grants from the National Academic Research Grant Funds (JSPS KAKENHI: 20H03913). The sponsor of the study had no role in the study design, data collection, analysis, or preparation of the manuscript. The authors report no other conflicts of interest in this work.

\section{References}

1. Waldorff FB, Petersen K, Vinther S, et al. Full journal publication of abstracts presented at the Nordic Congress of General Practice in 2009 and 2011. Scand J Prim Health Care. 2017;35(1):84-88. doi:10.1080/ 02813432.2017 .1288820
2. Egloff HM, West CP, Wang AT, et al. Publication rates of abstracts presented at the society of general internal medicine annual meeting. $J$ Gen Intern Med. 2017;32:673-678. doi:10.1007/s11606-017-39 90-5

3. Wachter RM, Goldman L. Zero to 50,000 - The 20th Anniversary of the hospitalist. N Engl J Med. 2016;375(11):1009-1011. doi:10.1056/ NEJMp1607958

4. Boonyasai RT, Lin YL, Brotman DJ, Kuo YF, Goodwin JS. Characteristics of primary care providers who adopted the hospitalist model from 2001 to 2009. J Hosp Med. 2015;10(2):75-82. doi: $10.1002 / \mathrm{jhm} .2269$

5. Puffer JC. The American Board of Family Medicine: celebrating 50 years of continuing transformation. J Am Board Fam Med. 2020;33 (Suppl):S69-S74. doi:10.3122/jabfm.2020.S1.190444

6. Japanese Medical Specialty Board homepage. About the specialty of General Medicine. Japanese. Available from: https://jmsb.or.jp/sogo/. Accessed January 21, 2021.

7. Watari T. The new era of academic hospitalist in Japan. J Gen Fam Med. 2020;21(2):29-30. doi:10.1002/jgf2.299

8. Do AND, Munchhof AM, Terry C, Emmett T, Kara A. Research and publication trends in hospital medicine. J Hosp Med. 2014;9 (3):148-154. doi:10.1002/jhm.2148

9. Wachter RM, Katz P, Showstack J, Bindman AB, Goldman L. Reorganizing an academic medical service: impact on cost, quality, patient satisfaction, and education. JAMA. 1998;279(19):1560-1565. doi:10.1001/jama.279.19.1560

10. O'Sullivan B, Coller BS. The research hospitalist: protocol enabler and protector of participant safety. Clin Transl Sci. 2015;8 (3):174-176. doi:10.1111/cts.12261

11. Japan Society for the Promotion of Science. Homepage. Available from: https://www.jsps.go.jp/english/index.html. Accessed January 21, 2021.

12. Friedman RH, Alpert JJ, Green LA. Strengthening academic generalist departments and divisions. J Gen Intern Med. 1994;9:S90-S98. doi:10.1007/BF02598123

13. Vineet Gupta MD, Neera Ahuja MD, Alana Sigmund MD, et al. State of research in adult hospital medicine: results of a national survey. J Hosp Med. 2019;14(4):207-211. doi:10.12788/jhm.3136

14. Reid MB, Misky GJ, Harrison RA, et al. Mentorship, productivity, and promotion among academic hospitalists. J Gen Intern Med. 2012;27(1):23-27. doi:10.1007/s11606-011-1892-5

15. Komagamine J, Yabuki T. Full-text publication rate of abstracts presented at the Japan Primary Care Association Annual Meetings (2010-2012): a retrospective observational study. BMJ Open. 2018;8: e021585. doi:10.1136/bmjopen-2018-021585
International Journal of General Medicine

\section{Publish your work in this journal}

The International Journal of General Medicine is an international, peer-reviewed open-access journal that focuses on general and internal medicine, pathogenesis, epidemiology, diagnosis, monitoring and treatment protocols. The journal is characterized by the rapid reporting of reviews, original research and clinical studies across all disease areas. The manuscript management system is completely online and includes a very quick and fair peer-review system, which is all easy to use. Visit http://www.dovepress.com/ testimonials.php to read real quotes from published authors. 